\title{
Determinantes de la pobreza en el Perú bajo el enfoque de activos
}

\author{
Determinants of poverty in Peru under the asset approach
}

\author{
Milagros Rosario Quispe Quispe ${ }^{1}$ \\ Richard Hernán Roca Garay
}

\section{RESUMEN}

El objetivo del presente trabajo es analizar los determinantes de la pobreza en el Perú bajo el enfoque de activos. A través del análisis del modelo de regresión logística se halla que el acceso a servicios básicos como agua potable, electricidad, servicios higiénicos y teléfono, reducen significativamente la probabilidad de ser pobre, así mismo, poseer título de propiedad de la vivienda que se habita y que ésta cuente con mayor número de habitaciones reduce la probabilidad de ser pobre. Mientras que no tener educación, jefe del hogar con lengua nativa quechua, aymara u otra lengua nativa y familias con mayor número de miembros aumenta la probabilidad de caer en pobreza.

Palabras clave: Pobreza; enfoque basado en los activos; Perú.

JEL: C25, I31, I3.

\footnotetext{
1 Economista por la Universidad Nacional Mayor de San Marcos, Lima, Perú. Máster en Economía por la Universidad Complutense de Madrid, España. Profesora auxiliar e investigadora, en la Facultad de Ciencias Económicas.

Correo-e: mquispeq@unmsm.edu.pe

2 Ex Decano de la Facultad de Ciencias Económicas en la Universidad Nacional Mayor de San Marcos. Profesor principal. Correo-e: rrocag@unmsm.edu.pe

(C) Los autores. Este artículo es publicado por Pensamiento Crítico de la Facultad de Ciencias Económicas, Universidad Nacional Mayor de San Marcos. Este es un artículo de acceso abierto, distribuido bajo los términos de la licencia Creative Commons Atribucion - No Comercia_Compartir Igual 4.0 Internacional. (http://creativecommons.org/licenses/by-nc-sa/4.0/) que permite el uso no comercial, distribución y reproducción en cualquier medio, siempre que la obra original sea debidamente citada.
} 


\section{ABSTRACT}

The objective of this paper is to analyze the determinants of poverty in Peru under the asset approach. Through the analysis of the logistic regression model, it is found that access to basic services such as drinking water, electricity, toilets and telephone, significantly reduce the probability of being poor, as well as owning the property of the dwelling that is inhabited and that it has a greater number of rooms reduces the probability of being poor. While having no education, head of household with native Quechua language, Aymara or other native language and families with larger number of members increases the likelihood of falling into poverty.

Keywords: Poverty; asset based approach; Peru.

JEL: C25, I31, I32.

\section{Introducción}

En el periodo 2009-2016, la economía en el Perú ha presentado una tendencia de crecimiento positiva, el Producto Bruto Interno (PBI) nacional creció a una tasa promedio de 4.7\%. Según el Banco Mundial (BM, 2017) el Perú fue una de las economías que destacó por presentar mayores niveles de crecimiento en América Latina y el Caribe, empero el tema principal si el crecimiento económico ha ayudado a mejorar la situación de peruanos que se encuentran en pobreza.

Así mismo, durante este periodo, la pobreza en el Perú se ha reducido 12.8 p.p. ${ }^{3}$, ha pasado de $33.5 \%$ en 2009 a $20.7 \%$ en 2016 . A pesar de este progreso significativo, la pobreza aún está presente en el Perú, según el Instituto nacional de Estadística e Informática (INEI, (2018, p. 41) "en el año 2017, el 21,7\% de la población del país, que equivale en cifras absolutas a 6 millones 906 mil personas, se encontraban en situación de pobreza, es decir, tenían un nivel de gasto inferior al costo de la canasta básica de consumo compuesto por alimentos y no alimentos. Al comparar estos resultados con el nivel obtenido en el año 2016, se observa que la pobreza aumentó en 1,0 p.p, que equivale a 375 mil personas pobres, más que en el año 2016". A pesar de los esfuerzos que se dado en la lucha contra la pobreza, el nivel aún es alto.

En este escenario, es fundamental analizar el fenómeno de la pobreza, por ello, el objetivo del presente estudio es analizar los determinantes

3 Puntos porcentuales. 
de la pobreza en el Perú bajo el enfoque de activos. Para este fin, se tomará como referente los años 2009, 2011 y 2016.

\section{Metodología}

\subsection{Indicadores sobre las condiciones de vida y pobreza}

La Encuesta Nacional de Hogares (ENAHO), es la investigación que permite al INEI, hacer el seguimiento de los indicadores sobre las condiciones de vida y pobreza en el Perú. La ENAHO es una encuesta especializada que se hace de forma continua desde el 2003, su ejecución tiene cobertura geográfica a nivel nacional.

Los objetivos de la ENAHO son: "(i) Generar indicadores mensuales que permitan conocer la evolución de la pobreza, del bienestar y de las condiciones de vida de los hogares, (ii) Efectuar diagnósticos (mensuales) sobres las condiciones de vida y pobreza de la población, (iii) Medir el alcance de los programas sociales en la mejora de las condiciones de vida de la población, (iv) Servir de fuente de información a instituciones públicas y privadas, así como a investigadores y (v) Permitir la comparabilidad con investigaciones similares, en relación a las variables investigadas". (ENAHO, 2009).

Los temas comprendidos son: características de la vivienda y el hogar, características de los miembros del hogar, salud, educación, empleo e ingresos, gastos del hogar, programas sociales, ingreso del trabajador independiente y dependiente, gobernabilidad, ingreso del trabajador agropecuario, democracia y transparencia. (ENAHO, 2016).

\subsection{Análisis de las variables consideradas en la investigación}

En el presente estudio, la variable que se intenta explicar, es decir, la variable dependiente es la pobreza monetaria que se expresa de forma binaria, esto es, toma el valor 1 si el hogar está en situación de pobreza y toma el valor cero si el hogar no está en situación de pobreza.

Las variables explicativas son:

Disponibilidad de agua: Las personas en situación de pobreza tiene por lo general menor acceso al agua potable que otros sectores sociales, 
esto incrementa el riesgo a enfermarse principalmente en población infantil, por ello, es de suma importancia disponer de este activo público básico ya que tiene un impacto muy significativo en la reducción de la pobreza.

Disponibilidad de servicios higiénicos: La ausencia de este activo aumenta el riesgo de contraer enfermedades infecciosas empeorando las condiciones de salud de las familias. Los hogares que no cuentan con este activo aumentan su vulnerabilidad frente a la pobreza, por esta razón, se incluye esta variable al presente estudio.

Disponibilidad de electricidad: Las familias con acceso a energía eléctrica pueden verse favorecidos en la generación de nuevas oportunidad o fuentes de trabajo, por ejemplo, permite que las mujeres y niñas tengan tiempo para buscar trabajo e ir a la escuela, esto incide significativamente en la disminución de la pobreza.

Disponibilidad de teléfono: Los teléfonos se han convertido en activos indispensables en nuestra sociedad, generan oportunidades laborales y actividades microempresariales, esto en la zona rural como zona urbana. Los beneficios obtenidos del uso este activo impactan significativamente en la disminución de pobreza.

La infraestructura es necesaria para disminuir la pobreza, la infraestructura aumenta la productividad de los trabajadores, permite la reducción de costos que afrontan los hogares, aumenta las oportunidades (BM, 1994). Muchos autores han analizado la relación de la infraestructura y la pobreza, por ejemplo, Reinikka y Svensson (1999), menciona que un hogar que no tiene acceso a agua, desagüe, electricidad y teléfono ve disminuidas sus posibilidades de inserción en los mercados. Ogun (2010) halló, a través de un modelo VAR estructural, que la infraestructura permitió disminuir significativamente la pobreza en las áreas urbanas de Nigeria. En el Perú el trabajo de Escobal y Torero (2003), señala que la geografía peruana impacta sobre la pobreza a través de la infraestructura pública.

Título de propiedad de la vivienda: Un medio para que un hogar pueda acceder al mercado crediticio puede ser a través de la posesión del título de propiedad de la vivienda, así, este activo se convertiría en un capital 
potencial que le permita generar ingresos. Al respecto, en el Perú el trabajo de De Soto (1986), menciona que la pobreza es ocasionada por la escasez de propiedad formal, obstaculizando que las personas puedan acceder al mercado crediticio.

Educación del jefe del hogar: La educación incide significativamente en la pobreza, el nivel de educación alcanzada por el individuo afecta positivamente su productividad y por consiguiente su capacidad de generación de ingresos, en tal sentido, los años de educación del jefe de familia es el capital humano que determina la condición de pobreza del hogar (Attansio y Székely, 2001). En el caso peruano, Benavides (2004) sostiene que a mayor educación en los padres mayor es el nivel de educación que presentan los hijos.

Idioma o lengua materna del jefe del hogar: El informe del BM señala que la desigualdad de ingresos se debe a "la raza, la región de origen, la educación de los padres y la ocupación de éstos en el momento del nacimiento" (BM, 2006, p. 5). Así, la raza o etnia está relacionada con la probabilidad de ser pobre. En el presente trabajo se usa como variable proxy a la lengua materna para analizar este hecho.

Número de miembros en el hogar: Diversos estudios realizados en el Perú y América Latina han detectado que cuando una familia cuenta muchos miembros aumenta la probabilidad de pobreza, específicamente, a mayor número de miembros en el hogar, mayor es la posibilidad de pobreza. Por ello, se incluye en la presente investigación esta variable considerando que los hogares peruanos se caracterizan por ser extensa.

Número de habitaciones de la vivienda: Cuando la familia cuenta con una vivienda que tiene una cantidad adecuada de habitaciones, de acuerdo al número de integrantes, sus condiciones de vida, además de ser favorables, aumentan. Al respecto, diversos estudios han encontrado una relación inversa entre el número de habitaciones en una vivienda y la pobreza.

En el tabla 1 se presenta un resumen de las variables que se incluirán en el modelo. 
Tabla 1

Descripción de variables del modelo

\begin{tabular}{ll} 
Variable & De scripción \\
De pe ndie nte & \\
\hline Condición de pobreza & 1 Pobre \\
& 0 No pobre \\
\hline Explicativas & \\
\hline Acceso_agua_potable & 1 Tiene acceso agua potable \\
& 0 Otros casos \\
\hline Acceso_servicios_higienicos & 1 Tiene acceso servicios higénicos \\
\hline Acceso_telefono & 0 Otros casos \\
\hline Acceso_alumbrado_electrico & 1 Tiene acceso teléfono \\
& 0 Otros casos \\
\hline Vivienda_titulo_propiedad & 1 Tiene acceso alumbrado electrico \\
& 0 Otros casos \\
\hline Num_habitaciones_vivienda & 1 Vivienda tiene título de propiedad \\
\hline Jefe_hogar_sin_educacion & 0 Otros casos \\
\hline Lengua_jefe_hogar & 1 Tiene educación \\
\hline & 0 Otros casos \\
\hline & 0 Otros casos \\
\hline & \\
\hline
\end{tabular}

Elaboración Propia

\subsection{El modelo de regresión con distribución logística}

Sea la variable binaria dependiente $y_{i}$ que se expresa de forma binaria con probabilidad de ocurrencia y no correncia $p$ y $1-p$, respectivamente.

Tomando la función logística, obtenemos el modelo Logit (Berkson, 1994):

$$
p\left(y_{i}=1\right)=\frac{1}{1+e^{-\alpha-\beta_{k} X_{k i}}}=\frac{e^{\alpha+\beta_{k} X_{k i}}}{1+e^{\alpha+\beta_{k} X_{k}}}
$$

Agregando el término de error:

$$
p\left(y_{i}=1\right)=\frac{e^{\alpha+\beta_{k} X_{k i}}}{1+e^{\alpha+\beta_{k} X_{k i}}}+\mu_{i}
$$


Derivando en (2) se obtiene el efecto marginal ante un cambio en la j-ésima variable independiente

\section{Marco teórico y antecedentes}

La pobreza corresponde a un problema que comprende varios aspectos, por ejemplo: acceso a agua potable, contar con habitación adecuada, ingresos percibidos, entre otros (GEEP, 2007). Por su parte, el BM (1990) define la pobreza como "la incapacidad para alcanzar un nivel de vida mínimo" (p. 26), mientras que la Comisión Económica para América Latina y el Caribe (CEPAL, 2001, p. 42) señala que "La pobreza es considerada un concepto eminentemente normativo, vinculado al bienestar de las personas, por lo que no existe una única noción de este fenómeno ni tampoco un método universal para medirlo". Podemos decir que hay un consenso en entender la pobreza como una condición en la cual uno o más personas tienen un nivel de bienestar inferior al mínimo necesario para la sobrevivencia.

De acuerdo con Ruggeri (2003), para realizar la medición de la pobreza, existen diversos enfoques, por ejemplo, Verdera (2007) expresa cuatro enfoques: Activos de los pobres, reconocimientos de derechos y capacidades, exclusión social y estructural. Adrianzen (2013) menciona que "no existe consenso en el ámbito internacional que permita obtener un único método de medición. Ya que la pobreza está relacionada con las distintas condiciones de insatisfacción y privación del ser humano" (p.44). En específico, se pueden distinguir cuatro enfoques básicos para medir la pobreza.

El enfoque de la pobreza subjetiva. De acuerdo a este, se debe tener en cuenta en la medición de la pobreza el índice de bienestar auto percibido, es decir, cómo percibe el individuo su propia situación. Este enfoque está relacionado con la "felicidad" o "satisfacción". Al respecto, la investigación de Ravallion y Lokshin (2002), encuentra que algunos rusos que sienten que son pobres no son clasificados como tal y los rusos que si son clasificados como pobres no se sienten de ese modo.

El enfoque de la pobreza multidimensional. Analiza la pobreza en términos de la capacidad y oportunidad que tiene la persona en diversas facetas. Al respecto, el Oxford Poverty and Human Development Initiative 
(OPHI) ha desarrollado el Índice de Pobreza Multidimensional con base a educación, salud y condiciones de vida (Alkire \& Santos, 2010).

El enfoque de necesidades básicas insatisfechas. De acuerdo a este enfoque, se estudia la pobreza en términos del acceso a: educación, vivienda, consumo, agua, saneamiento, electricidad y comunicación.

El enfoque de la pobreza monetaria. La pobreza puede ser medida por el nivel de ingreso o por el nivel de gasto, así, una persona es pobre en el sentido monetario si su ingreso, diario o mensual, es menor respecto a un ingreso determinado. Así mismo, es pobre monetario un individuo cuando su gasto per cápita es menor respecto del valor de una canasta de bienes básico para el consumo.

En el Perú se considera a una persona pobre bajo el enfoque monetario, es decir, es pobre en términos monetarios un peruano cuyo gasto per cápita mensual no supera el costo de una canasta de alimentos y no alimentos. La entidad que se encarga de otorgar la información sobre la pobreza es el INEI, para ello se basa en la Encuesta Nacional de Hogares (ENAHO), en su informe "Evolución de la Pobreza Monetaria 2007-2016" expresa que la línea de pobreza, expresado valor monetario, se contrasta el gasto per cápita mensual de un hogar para determinar si está en situación de pobreza o no (INEI, 2018).

\subsection{Enfoque de la pobreza basado en los activos}

El enfoque basado en los activos fija su atención en los activos públicos, en los activos privados y en los activos sociales de los hogares. Siegel (2005) postula que los activos de los hogares son considerados conductores del crecimiento sostenible y la reducción de la pobreza. Bajo esa misma línea, específica que los pobres son pobres en activos porque tienen pocos activos.

Ahora bien, uno de los principales factores de la pobreza en América Latina es la desigualdad que existe en la distribución de los activos, al respecto Attanasio y Székely (2001, p. 2) señala que "la pobreza en América Latina o, al menos, el exceso de pobreza viene dado por el nivel de ingresos en la región [...] la desigualdad de ingresos en la región es en 
gran medida, un reflejo de una distribución muy desigual de los activos generadores de ingresos".

Siguiendo con el enfoque de los activos para analizar la pobreza, Attanasio y Székely (2001) plantean que el ingreso per cápita por familia, indicador de bienestar utilizado tradicionalmente en América Latina, puede expresarse como:

$$
y_{i}=\frac{\left(\sum_{i=1}^{j} \sum_{a=1}^{l} A_{a, 1} R_{a, i} P_{a}\right)+\sum_{i=1}^{k} T_{i}}{n}
$$

Esta función de ingresos es una combinación de cuatro elementos donde:

$\mathrm{A}_{\mathrm{a}, 1}$ : stock del activo del individuo ${ }^{\mathrm{i}}$.

$\mathrm{R}_{\mathrm{a}, \mathrm{i}}$ : tasa.

$\mathrm{P}_{\mathrm{a}}$ : valor de mercado por unidad.

$\mathrm{T}_{\mathrm{i}}$ : transferencia perteneciente al individuo ${ }^{\mathrm{i}}$

j: cantidad de personas que generan ingresos en el hogar al que pertenece el individuo $\mathrm{i}$.

l: distintos activos.

k: cantidad de personas en el hogar que obtiene ingresos por transferencias.

Attanasio y Székely (2001) señalan que los años de estudio de un individuo sólo se traducirá en ingresos si existe su participación de mercado de trabajo (es esto es cuando $R>0$ en la ecuación 1), el capital físico se convertirá en ingreso cuando el retorno del activo se haga efectivo y el sociales capital tendrá un efecto positivo cuando se utilicen las relaciones sociales para generar ingresos. En resumen, el ingreso que puede obtener el individuo dependerá del uso de sus activos en el mercado.

Respecto al stock de activos, estos pueden ser de tres tipos, el capital humano, el capital físico y el capital social. El capital humano, es el con- 
junto de habilidades que necesita una persona para producir un bien o servicio, las variables más utilizadas para su cuantificación son los años educación, experiencia laboral, potencial, diferencia en género, tamaño del hogar, entre otros. El capital físico, que incluye el capital físico privado y el capital físico social, el capital físico privado, referido a propiedades, tierras, ganados, activo financiero o activos empresariales, entre otros. El capital físico social, referido al acceso a servicios de agua, electricidad, sanidad, otros).

Tanto el capital físico como el capital humano son factores determinante para la pobreza, y hacen menos vulnerables a las familias ante los riesgos de caer en ella, al respecto el BM menciona que la disponibilidad de activos desempeña con frecuencia un papel importante y para ello menciona un ejemplo "En China, la falta de capital físico es un factor determinante de la pobreza tanto crónica como transitoria, pero el tamaño del hogar y el nivel de instrucción del jefe del mismo determinan la probabilidad de pobreza crónica pero no transitoria" (BM, 2001, p. 43).

El capital social, referido a normas y redes sociales que facilitan la acción colectiva entre las personas, el núcleo principal del capital social son los valores tales como la solidaridad, la confianza, la honestidad, la reciprocidad. Las redes sociales, institucionales y organizacionales, facilitan la interacción social y el desarrollo de las sociedades. En tal sentido, el Programa de las Naciones Unidas para el Desarrollo (PNUD, 2002, p. 8) define al capital social como las "reglas, valores, organizaciones y recursos sociales que permiten que las personas se organicen y logren combinar los otros factores, recursos o capitales".

\subsection{Los activos de los pobres, caso peruano}

El estudio de Torero, Escobal y Saaedra (2001), encontró que la infraestructura agua, desagüe, electricidad y teléfono impactan significativamente sobre la pobreza, destacando, la tenencia de teléfono en la zona urbana. Posteriormente, el trabajo de Escobal y Torero (2004) analiza el efecto que ha tenido la infraestructura pública en las zonas rurales, encontraron que un mayor acceso a servicios de infraestructura rural impacta positivamente en la reducción de la pobreza. 
Villacorta (2011, p. 57), constató que los pobres poseen recursos que interactúan con las oportunidades que derivan del mercado, del Estado y de la sociedad. "Estos recursos son los activos, enfoque que va más allá del factor ingresos y necesidades básicas insatisfechas como criterios que determinan la condición de pobreza. Bajo este enfoque, si bien los ingresos son un factor clave de los activos de los pobres, estos dependen de otros factores que determinarán en definitiva la posición social y las posibilidades de un hogar de permanecer o salir de la pobreza. Por ejemplo, el nivel de instrucción de los padres, la propiedad sobre algún activo como (la vivienda, la tierra, un vehículo, equipamiento, etc.), el número de miembros del hogar y la participación en organizaciones o redes son factores que, interactuando con las condiciones sociales imperantes, inciden sobre el nivel socioeconómico y posición social de los hogares".

Webb (2013) halló que familias con viviendas inadecuadas sin servicios de saneamiento, presentaron bajos niveles de productividad. Expresa que el despegue en el ámbito rural del Perú sería por la difusión de la telecomunicación.

Aparicio, Jaramillo y San Román (2011) hallan que los distintos tipos de infraestructura tales como: comunicación, luz eléctrica, agua y saneamiento, son activos que permiten generar ingresos en las familias. También, luego de hacer un análisis transversal, encuentran que "la probabilidad de ser pobre pasa de $45 \%$ cuando no se tiene infraestructura alguna en el hogar a 12\% cuando se tienen las cuatro infraestructuras", (p.41).

\subsection{Evolución de la pobreza}

Como se puede observar en la figura 1, del 2009 al 2016 la pobreza en el Perú se ha reducido en 12.8 p.p., ha pasado de 33.5\% en 2009a 20.7\% en 2016. Respecto a la pobreza extrema, en este periodo también ha decrecido, ha pasado de $9.5 \%$ en 2009 a $3.8 \%$ en 2016, lo que significa una disminución de 5.7p.p.

En cuanto a la pobreza en la zona urbana y en la zona rural, las figuras 2 y 3 muestran que la pobreza rural es mayor que la pobreza urbana, lo que significa que la desigualdad aún está presente. Durante el periodo de análisis la pobreza urbana disminuyó en 7.4 p.p. al pasar de $21.3 \%$ en 2009 a $13.9 \%$ en 2016, mientras que la pobreza rural disminuyóen22.9 
p.p. al pasar de $66.7 \%$ a $43.8 \%$. En cuanto a la pobreza extrema, ésta cayó más en el área rural pasando de $29.8 \%$ a $13.2 \%$, lo que significa una reducción de 16.6 p.p.

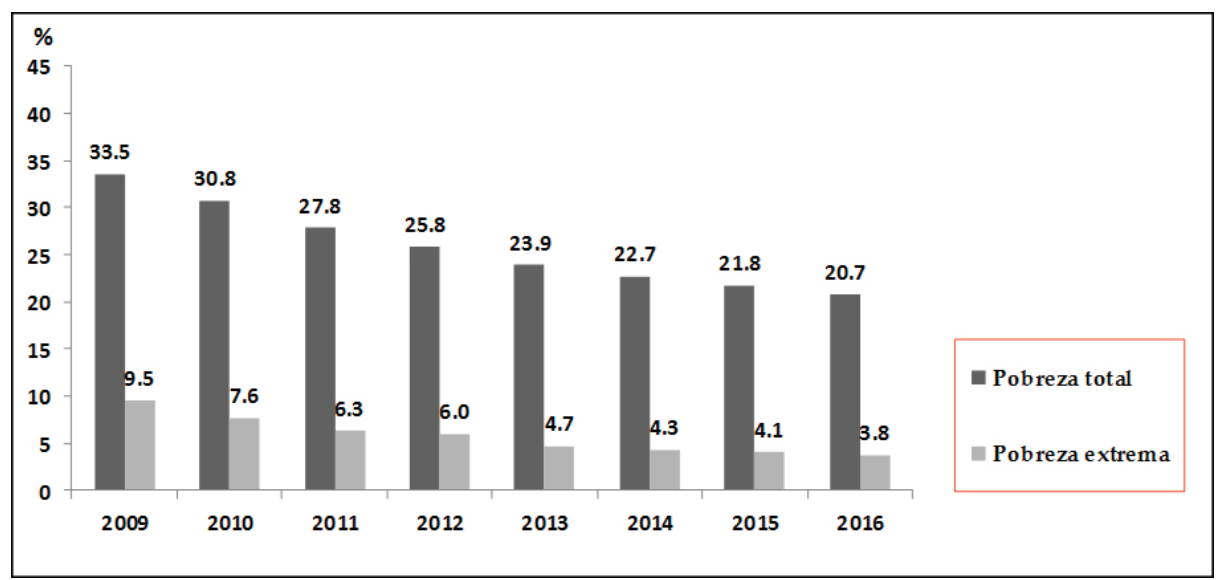

Figura 1. Pobreza en el Perú 2009-2016 (En porcentaje)

Elaboración: Propia a partir de la ENAHO 2009-2016 (INEI)

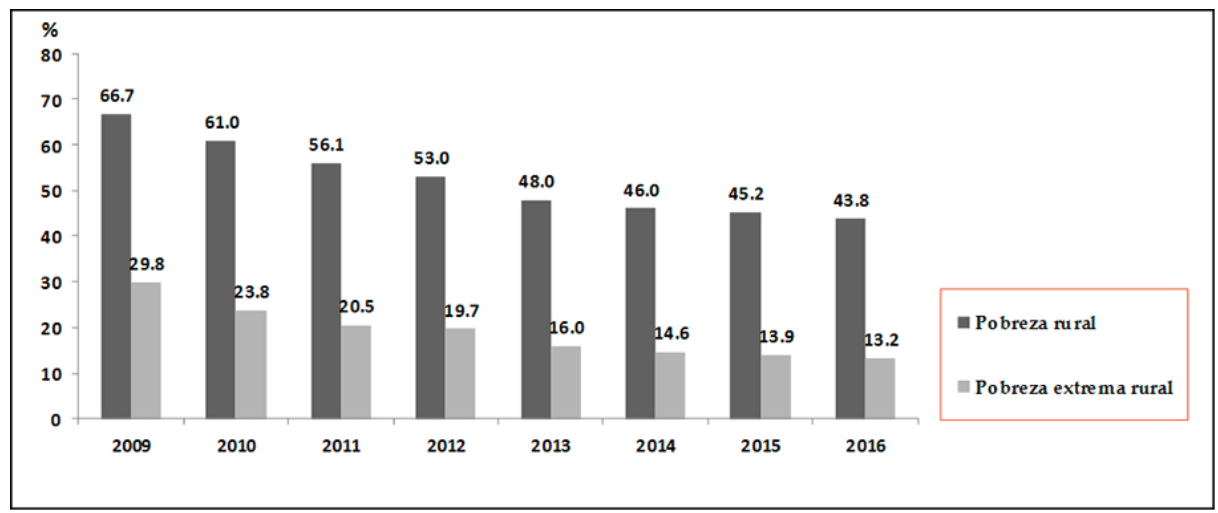

Figura 2. Pobreza rural en el Perú 2009-2016 (En porcentaje)

Elaboración: Propia a partir de la ENAHO 2009-2016 (INEI) 


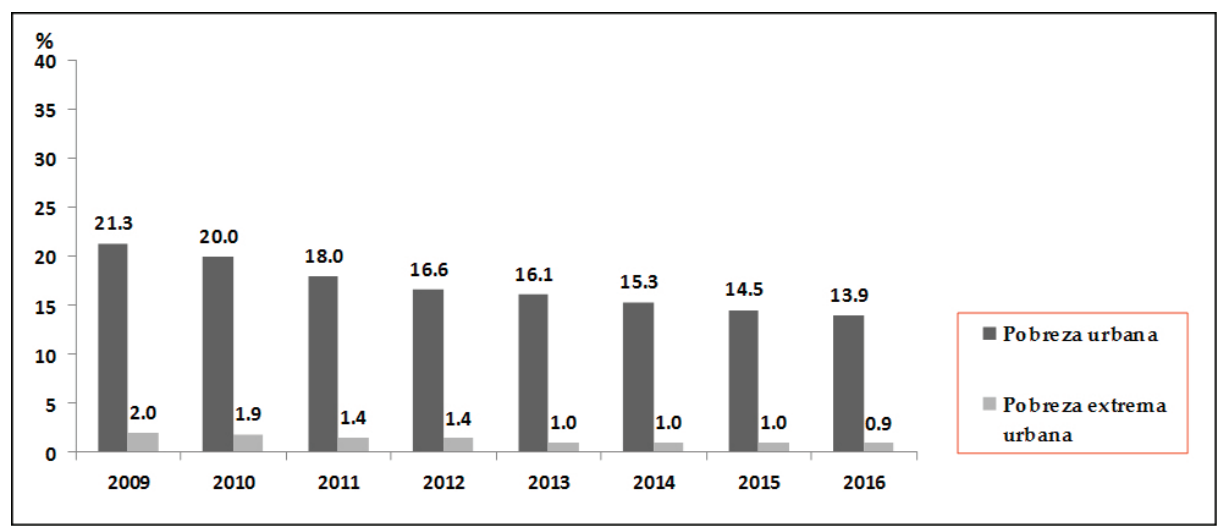

Figura 3. Pobreza urbana en el Perú 2009-2016 (En porcentaje) Elaboración: Propia a partir de la ENAHO 2009-2016 (INEI)

\subsection{Evolución de los servicios básicos}

Durante el periodo 2007-2017 el acceso a los servicios básico de la población pobre ha aumentado. En la figura 4 se observa que el acceso a los servicios básicos ha aumentado notoriamente, con excepción del saneamiento.

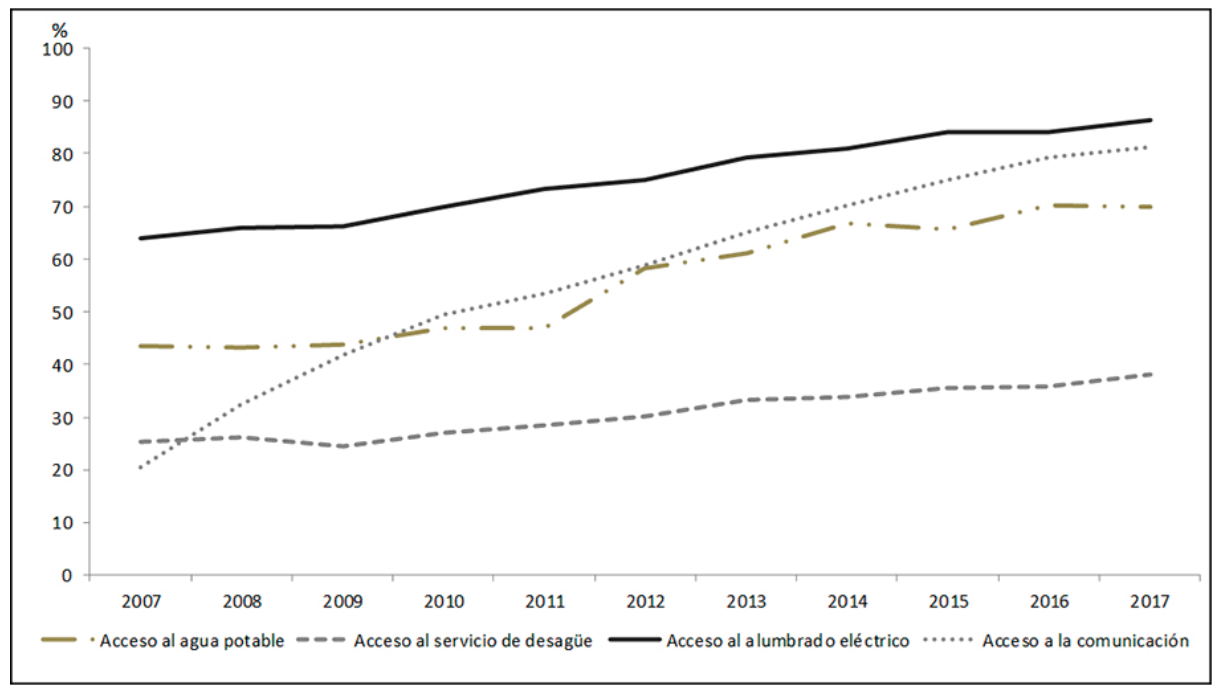

Figura 4. Acceso a servicios básico en la población pobre, Perú 2007-2017 Elaboración: Propia a partir de la ENAHO 2009-2016 (INEI) 
En 2017 el 61.9\% de peruanos pobres no accede a servicio de saneamiento. Cabe notar que se registró un incremento de a acceso a agua potable de 2007 a 2016, empero se redujo ligeramente en 2017. Respecto al alumbrado eléctrico y la comunicación, se incrementaron sobre el $80 \%$.

\section{Resultados}

Con los datos de la ENAHO, se corrieron modelos de regresión logística a nivel nacional y los efectos marginales para los años 2009, 2011 y 2016, en la tabla 2 se muestra los resultados ${ }^{4}$ :

Tabla 2

Modelo de regresión logística - Estimaciones de los determinantes de la pobreza, Perú 2009-2011-2016

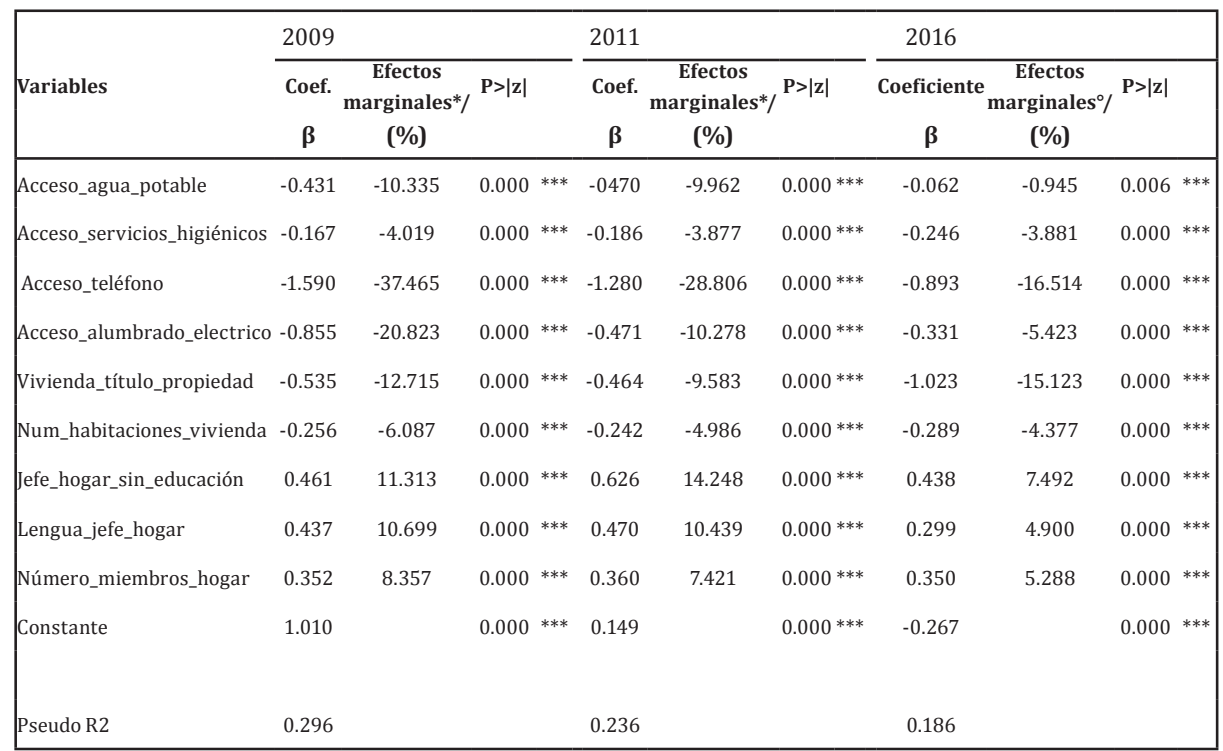

*/ Efecto marginal para el cambio discreto de la variable dummy al pasar de 0 a 1 .

*** Significativo al 1\%, ** significativo al 5\%, *significativo al 10\%.

Elaboración: Propia a partir de la ENAHO 2009-2011-2016 (INEI).

Como se observa en la tabla 2 , todas las variables independientes son significativas en cada periodo de estudio a un nivel de confianza de 99\%,

4 Ver en anexoslos resultados de las regresiones para los años 2009, 2011 y 2016 y los efectos marginales. 
lo que demuestra que el acceso a agua potable, a servicios higiénicos, a teléfono y a alumbrado eléctrico, son determinantes muy significativos para reducir la pobreza, pues sus coeficientes son intuitivamente correctos y significativos en cada periodo de estudio.

El acceso a agua potable reducía la probabilidad de ser pobre alrededor de 10\%, en 2009 y 2011 y alrededor de 1\% en 2016. El acceso a los servicios higiénicos reducía la probabilidad de ser pobre alrededor de $4 \%$, en los tres años de análisis. La reducción de la incidencia del acceso a agua potable sobre la pobreza se podría explicarse porque 2009 existía una marcada presencia de la falta de acceso a agua potable, principalmente en las áreas rurales, Aparicio et. al. (2011). Luego se dieron avances, Vásquez (2013) expresa que millones de peruanos dejaron de tener la carencia de acceso a agua potable.

Un comportamiento similar se encuentra en el alumbrado eléctrico, esté reducía la probabilidad de ser pobre alrededor de 21\%, en 2009, 10\% en 2011 y 5\% en 2016. Esto se podría explicar porque la infraestructura eléctrica habría aumentado en este periodo de estudio.

Respecto a la comunicación, se tiene que el acceso al teléfono es la variable que más incide sobre la probabilidad de reducir la pobreza, de todas las variables incluidas en el modelo.

Presenta mayor efecto marginal, pues, reducía la probabilidad de ser pobre alrededor de 37\%, en 2009, 29\% en 2011 y 17\% en 2016. Similar hallazgo se encuentra en trabajo de la consultora Apoyo Consultoría (2010) y el trabajo de Aparicio et. al. (2011), ambos estudios hallan impacto significativo respecto a la tenencia de teléfonos móviles.

Tener el título de propiedad de la casa en que se habita, disminuía la probabilidad de ser pobre alrededor de 13\%, en 2009, $10 \%$ en 2011 y $15 \%$ en 2016. Esto quiere decir, que la ausencia de esta variable es un problema existente en el Perú, a pesar de un crecimiento significativo del sector inmobiliario el precio de las viviendas se han incrementado notoriamente, al respecto Westreicher (2013), menciona que la demanda insatisfecha, aproximadamente $90 \%$ de peruanos, no tenía más de 30.000 dólares acceder a una vivienda. 
Se sabe que la educación es una variable muy importante para atacar la pobreza, en el presente estudio se halla que, no tener educación aumentaba la probabilidad de ser pobre alrededor de 11\%, en $2009,14 \%$ en 2011 y 7\% en 2016. Estos resultados confirman que no tener educación impacta significativamente en el incremento de la pobreza, siendo consistente además con los resultados de Villacorta (2011), "Los años de estudio promedio de los integrantes del hogar mayores de 14 años, es otro factor que reduce considerablemente la probabilidad de ser pobre, es así, que coincidiendo con otros estudios, la educación como capital humano es un activo que permite el acceso a nuevas oportunidades laborales, mejorando el bienestar de los pobres" (p. 8).

Cabe notar que, hogares con numerosas habitaciones disminuía la probabilidad de caer en pobreza alrededor de 6\% en 2009, 5\% en 2011 y 4\% en 2016. En contraste, en el Perú, hablar una lengua materna sea está quechua, aymara u otra lengua nativa, aumentaba la probabilidad de caer en pobreza alrededor de $11 \%$ en $2009,10 \%$ en 2011 y 5\% en 2016, así mismo, hogares con muchos miembros aumentaba la probabilidad de ser pobre alrededor de 8\% en 2009, 7\% en 2011 y 5\% en 2016.

\section{Conclusiones y recomendaciones}

Son los determinantes más significativos sobre la probabilidad de disminuir la pobreza, accederá agua potable, a servicios higiénicos, a alumbrado eléctrico y principalmente el acceso a teléfono.

Poseer el título de propiedad de la casa, es una determinante relevante sobre la probabilidad de disminuir la pobreza, en 2016 tenía un efecto marginal significativamente alto (15\%).

Los resultados hallados muestran que la educación incide significativamente sobre la probabilidad de reducir la pobreza.

Hablar una lengua materna sea está quechua, aymara u otro idioma nativo incrementa la probabilidad de ser pobre.

Familias con muchos miembros incrementaba la probabilidad de ser pobre, en contraste, familias con mayor número de habitaciones reduce la probabilidad de pobreza. 
Dado lo precedente, se recomienda lo siguiente:

Dado que es el acceso a servicios básicos, impacta significativamente sobre la probabilidad de reducir la pobreza, se debe priorizar en impulsar el desarrollo de la infraestructura de servicios de agua potable, servicios higiénicos, alumbrado eléctrico y telefonía, en todo el Perú.

Respecto a la propiedad de la vivienda, permitir que más peruanos, accedan a la casa propia otorgando diversas facilidades.

En cuanto a la educación, crear políticas educativas dirigidas, a incrementar la oferta educativa de calidad y mejorar la oferta educativa que ya existe.

Incentivar programas educativos, dirigidos a peruanos con lengua nativa, para la enseñanza del castellano como segunda lengua.

Se recomienda implementar diversos programas de planificación familiar, a fin de, evitar que familias muy numerosas caigan en pobreza.

Finalmente, se recomienda dar facilidades para que más peruanos puedan acceder a la construcción de su vivienda.

\section{Referencias}

Adrianzén, Carlos (2013).Crecimiento y pobreza en el Perú: 2001-2011. Revista de Economía y Derecho, p. 35-66.Recuperado de https://repositorioacademico. upc.edu.pe/handle/10757/550861

Alkire, Sabina, y Santos, María Emma (2010).Acute Multidimentional Poverty: A New Index for Developing Countries. United Nations development programme human development report office background paper. Working Paper № 38 . 139p.doi: org/10.1016/j.worlddev.2014.01.026

Aparicio, Carlos; Jaramillo, Miguel y San Román, Cristina. (2011). Desarrollo de la infraestructura y reducción de la pobreza: El caso peruano. Lima: Consorcio de Investigación Económica y Social. 67p.Recuperado de https://www.cies. org.pe/sites/default/files/investigaciones/desarrollo-de-la-infraestructura-y-reduccion-de-la-pobreza_0.pdf

Apoyo Consultoría (2010). El Impacto de las Telecomunicaciones en el Desarrollo: El Caso de la Telefonía Móvil en el Ámbito Rural. Lima, Perú. 
Attanasio, Orazio y Székely, Miguel (2001).Going beyond income: Redefining poverty in Latin America. En: Attanasio, O., y Székely, M. (eds.). Potrait of the Poor: An Assets-Based Approach. Washington D.C.: Inter-American Development Bank, p. 1-44. [sin doi asignado]

Banco Mundial (2017).Perú Systematic Country Diagnostic. Wordd Bank Group. February 2017.184p.

Banco Mundial (2006). Motores de crecimiento rural sostenible y reducción de la pobreza en Centroamérica. Estudio caso de Nicaragua, Documento de trabajo $\mathrm{N}^{\circ} 23$. Banco Mundial, Proyecto Ruta. 82p.

Banco Mundial BM (2001). Informe sobre el Desarrollo Mundial 2000/2001. Lucha contra la Pobreza. Washington D.C.: Mundi-Prensa Libros, S.A., 335p.

Banco Mundial (1994). Informe sobre el Desarrollo Mundial 1994: Infraestructura y Desarrollo.Washington: Oxford University Press.266p.

Banco Mundial (1990).World Development Report: Poverty. Washington DC, Estados Unidos, 260p.

Berkson, Joseph (1944). Application of the logistic function to bio-assay. Journal of the American Statistical Association, vol. 39, no 227, p. 357-365.

Comisión Económica para América Latina y el Caribe (2001). 2000/2001 Panorama social de América Latina. Publicación de las Naciones Unidas LC/G.2138P, Santiago de Chile, Chile, 271p.

De Soto, Hernando (1986). El Otro Sendero. Lima: Instituto Libertad y Democracia.15p.Recuperado de https://www.elcato.org/pdf_files/PrologoVargas-Llosa.pdf

Escobal, Javier y Máximo Torero (2003). Adverse geography and differences in welfare in Peru. Discussion Paper $\mathrm{N}^{\circ}$ 2003/73 United Nations University. World Institute for Development Economics Research, p.17-122.Recuperado de https://www.grade.org.pe/publicaciones/591-adverse-geography-anddifferences-in-welfare-in-peru/

Grupo de Expertos en Estadísticas de Pobreza GEEP (2007). Compendio de mejores prácticas en la medición de la pobreza. Grupo de Río, Santiago de Chile, Cepal, 2007. 139p. Recuperado de https://www.cepal.org/ilpes/noticias/ paginas/2/40352/rio_group_compendium_es.pdf

Instituto Nacional de Estadística e Informática (2017). Evolución de la Pobreza Monetaria 2007-2016. Informe Técnico, Lima, 181p. Recuperado de https:// www.inei.gob.pe/media/MenuRecursivo/publicaciones_digitales/pobreza2016.jpg 
Instituto Nacional de Estadística e Informática (2018). Evolución de la Pobreza Monetaria 2007-2017. Informe Técnico, Lima, 181p.Recuperado de https:// www.inei.gob.pe/media/MenuRecursivo/publicaciones_digitales/Est/ Lib1533/index.html

Novales, Alfonzo. (1996). Econometría. Segunda edición, Editorial: McGraw-Hill. $676 \mathrm{p}$.

Ogun, TP (2010). Infraestructure and poverty reduction: Implications for urban development in Nigeria. United Nations University - World Institute for Development Economics Research, Working Paper № 2010/43.Vol. P. 43:1-18. doi. org/10.1007/s12132-010-9091-8

Programa de las Naciones Unidas para el Desarrollo (2002), "Informe sobre desarrollo humano Perú, Aprovechando las potencialidades", Lima, 275p.

Ravallion, Martin y Lokshin, Michael (2002).Self-rated economic welfare in Russia. European Economic Review, 46(8), p. 1453-1473. Recuperado de https:// ssrn.com/abstract $=326643$

Reinikka, Ritva y Svensson, Jakov (1999). How inadequate provision of public infrastructure and services affect private investment. World Bank, Working Paper № 2262, 18p. doi.org/10.1596/1813-9450-2262

Ruggeri, Caterina; Saith, Ruhi., y Stewart, Frances (2003). Does It Matter That We don't Agree on The Definition of Poverty? A Comparison of Four Approaches. QEH Working Paper Series 107, mayo, 41p.Recuperado de https://www. ophi.org.uk/wp-content/uploads/ssRuggeri-Laderchi-Saith-Stewart-2003. pdf

Siegel, Paul (2005). Using an Asset-Based Approach to Identify Drivers of Sustainable Rural Growth and Poverty Reduction in Central America. A Conceptual Framework.30p. Recuperado de http://documents.worldbank.org/curated/en/307691468743185566/Using-an-asset-based-approach-to-identifydrivers-of-sustainable-rural-growth-and-poverty-reduction-in-CentralAmerica-a-conceptual-framework

Spicker, Paul; Álvarez Leguizamón, Sonia, y Gordon, David (2009). Pobreza un glosario internacional. (No. 3). e-libro, Corp. 320p.

Torero, Máximo; Escobal, Javier y Saavedra, Jaime. (2001). Distribution, access and complementarity: Capital of the poor in Peru. En: Attanasio, O., y Székely, M. (eds.). Potrait of the Poor: An Assets-Based Approach. Washington D.C.: Inter-American Development Bank, p. 209-240.Recuperado de https://www. grade.org.pe/publicaciones/385-distribution-access-and-complementarity-capital-of-the-poor-in-peru/ 
Vásquez, Enrique (2013). Las políticas y programas sociales del gobierno de Ollanta Humala desde la perspectiva de la pobreza multidimensional. Centro de Investigaciones de la Universidad del Pacífico, Documento de Discusión, 176p. doi:http://hdl.handle.net/11354/429

Verdera, Francisco (2007). Enfoques de la pobreza en la pobreza en el Perú: un análisis de sus causas y de las políticas para enfrentarla. Serie Análisis Económico, 24, IEP. doi: http://biblioteca.clacso.edu.ar/clacso/coediciones/ 20130829033132/02enfoque.pdf

Villacorta, Mirlena (2011). Perú: Determinantes de la Pobreza, 2009. Centro de Investigación y Desarrollo del Instituto Nacional de Estadística e Informática (INEI). 74p. doi: http://textos.pucp.edu.pe/texto/PERU-DETERMINANTESDE-LA-POBREZA-2009

Webb, Richard (2013). Conexión y Despegue Rural. Lima: Universidad San Martín de Porres. 271p. Recuperado de https://www.edugestores.pe/docs/conexion-y-despegue-rural/

Westreicher, G. (2013). El 90\% de la demanda inmobiliaria 'insatisfecha' no puede pagar más de US\$30 mil por vivienda, Gestión, 6 de junio. [sindoi asignado] 


\section{ANEXOS}

Anexo1

Modelo de regresión logística - Estimación de los determinantes de la pobreza, Perú 2009

Logistic regression

Log likelihood $=-35836.303$

$\begin{array}{llr}\text { Number of obs } & = & 75208 \\ \text { LR chi2(9) } & = & 30116.39 \\ \text { Prob > chi2 } & = & 0.0000 \\ \text { Pseudo R2 } & = & 0.2959\end{array}$

\begin{tabular}{r|rrrrrr}
\hline situacion_pobreza & Coef. & Std. Err. & $z$ & P >|z| & [95\% Conf. Interval] \\
\hline acceso_agua_potable & -.4313875 & .0212372 & -20.31 & 0.000 & -.4730117 & -.3897633 \\
acceso_servicios_higienicos & -.1674997 & .0241712 & -6.93 & 0.000 & -.2148743 & -.1201251 \\
acceso_telefono & -1.589525 & .0212248 & -74.89 & 0.000 & -1.631124 & -1.547925 \\
acceso_alumbrado_electrico & -.8546608 & .0253556 & -33.71 & 0.000 & -.9043569 & -.8049648 \\
vivienda_titulo_propiedad & -.5352969 & .0191161 & -28.00 & 0.000 & -.5727638 & -.49783 \\
num_habitaciones_vivienda & -.2562783 & .005888 & -43.53 & 0.000 & -.2678185 & -.244738 \\
jefe_hogar_sin_educacion & .4607869 & .0607242 & 7.59 & 0.000 & .3417696 & .5798043 \\
lengua_jefe_hogar & .4369011 & .0423059 & 10.33 & 0.000 & .3539831 & .5198191 \\
numero_miembros_hogar & .3518385 & .0044658 & 78.78 & 0.000 & .3430856 & .3605914 \\
\hline _cons & 1.010251 & .034198 & 29.54 & 0.000 & .9432238 & 1.077277 \\
\hline
\end{tabular}

\section{Anexo2}

Modelo de regresión logística - Estimación de los determinantes de la pobreza, Perú 2009, (efectos marginales)

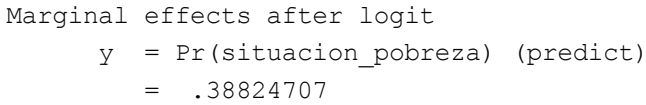

\begin{tabular}{|c|c|c|c|c|c|c|c|}
\hline variable & $d y / d x$ & Std. Err. & z & $\mathrm{P}>|\mathrm{z}|$ & $95 \%$ & C.I. & $\mathrm{X}$ \\
\hline acceso e* & -.103346 & .00511 & -20.23 & 0.000 & -.113356 & -.093336 & .633004 \\
\hline acceso s * & -.0401913 & .00585 & -6.87 & 0.000 & -.051662 & -.028721 & .802867 \\
\hline acces no* & -.3746469 & .00464 & -80.81 & 0.000 & -.383734 & -.36556 & .663453 \\
\hline $\operatorname{acces} \sim \mathrm{co}^{*}$ & -.2082286 & .00617 & -33.75 & 0.000 & -.22032 & -.196137 & .784012 \\
\hline vivien d* & -.1271526 & .00451 & -28.19 & 0.000 & -.135993 & -.118312 & .545088 \\
\hline num_ha a & -.060869 & .00139 & -43.77 & 0.000 & -.063595 & -.058143 & 3.70019 \\
\hline jefe_h n* & .1131305 & .01516 & 7.46 & 0.000 & .083417 & .142844 & .025024 \\
\hline lengua r* & .1069945 & .01054 & 10.15 & 0.000 & .086339 & .12765 & .054648 \\
\hline numero r & .0835656 & .00106 & 78.63 & 0.000 & .081483 & .085649 & 5.32036 \\
\hline
\end{tabular}

(*) $d y / d x$ is for discrete change of dummy variable from 0 to 1 


\section{Milagros Rosario QuisPe QuisPe, RichaRd HERnán Roca GaRAY}

\section{Anexo3}

Modelo de regresión logística - Estimación de los determinantes de la pobreza, Perú 2011

Logistic regression

Log likelihood $=-41062.728$

$\begin{array}{llr}\text { Number of obs } & = & 84588 \\ \text { LR chi2(9) } & = & 25356.73 \\ \text { Prob > chi2 } & = & 0.0000 \\ \text { Pseudo R2 } & = & 0.2359\end{array}$

\begin{tabular}{r|rrrrrr}
\hline situacion_pobreza & Coef. & Std. Err. & z & P $>|z|$ & [95\% Conf. Interval] \\
\hline acceso_agua_potable & -.4700276 & .0200913 & -23.39 & 0.000 & -.5094058 & -.4306494 \\
acceso_servicios_higienicos & -.1858323 & .0193879 & -9.58 & 0.000 & -.2238319 & -.1478326 \\
acceso_telefono & -1.28011 & .0209433 & -61.12 & 0.000 & -1.321158 & -1.239062 \\
acceso_alumbrado_electrico & -.4712679 & .0244739 & -19.26 & 0.000 & -.5192359 & -.4233 \\
vivienda_titulo_propiedad & -.4639812 & .0180495 & -25.71 & 0.000 & -.4993577 & -.4286048 \\
num_habitaciones_vivienda & -.2419516 & .0056565 & -42.77 & 0.000 & -.2530381 & -.230865 \\
jefe_hogar_sin_educacion & .6260711 & .0518774 & 12.07 & 0.000 & .5243933 & .7277489 \\
lengua_jefe_hogar & .4698694 & .0378374 & 12.42 & 0.000 & .3957095 & .5440292 \\
numero_miembros_hogar & .3601073 & .0042729 & 84.28 & 0.000 & .3517326 & .368482 \\
\hline
\end{tabular}

\section{Anexo4}

Modelo de regresión logística - Estimación de los determinantes de la pobreza, Perú 2011, (efectos marginales)

$$
\begin{aligned}
\text { Marginal effects after logit } & \\
y & =\operatorname{Pr}(\text { situacion_pobreza) (predict) } \\
& =.29045153
\end{aligned}
$$

\begin{tabular}{|c|c|c|c|c|c|c|c|}
\hline variable & $d y / d x$ & Std. Err. & z & $\mathrm{P}>|\mathrm{z}|$ & $95 \%$ & C.I. & $\mathrm{X}$ \\
\hline acceso e* & -.09962 & .00435 & -22.88 & 0.000 & -.108154 & -.091086 & .661146 \\
\hline acceso s* & -.0387747 & .00409 & -9.48 & 0.000 & -.046794 & -.030756 & .666005 \\
\hline acces no* & -.2880569 & .00486 & -59.30 & 0.000 & -.297578 & -.278536 & .755072 \\
\hline $\operatorname{acces} \sim \mathrm{co}^{\star}$ & -.1027837 & .00562 & -18.30 & 0.000 & -.11379 & -.091777 & .826689 \\
\hline vivien $d^{\star}$ & -.0958329 & .00371 & -25.81 & 0.000 & -.103111 & -.088555 & .522651 \\
\hline num_ha a & -.0498637 & .00115 & -43.30 & 0.000 & -.052121 & -.047607 & 3.73753 \\
\hline jefe_h n* & .1424781 & .01265 & 11.26 & 0.000 & .117686 & .16727 & .027356 \\
\hline lengua r* & .1043866 & .00892 & 11.70 & 0.000 & .086905 & .121869 & .056131 \\
\hline numero r & .0742143 & .00088 & 84.66 & 0.000 & .072496 & .075932 & 5.14138 \\
\hline
\end{tabular}

(*) dy/dx is for discrete change of dummy variable from 0 to 1 
Pensamiento Crítico Vol. 24. $\mathbf{N}^{\circ} 1$

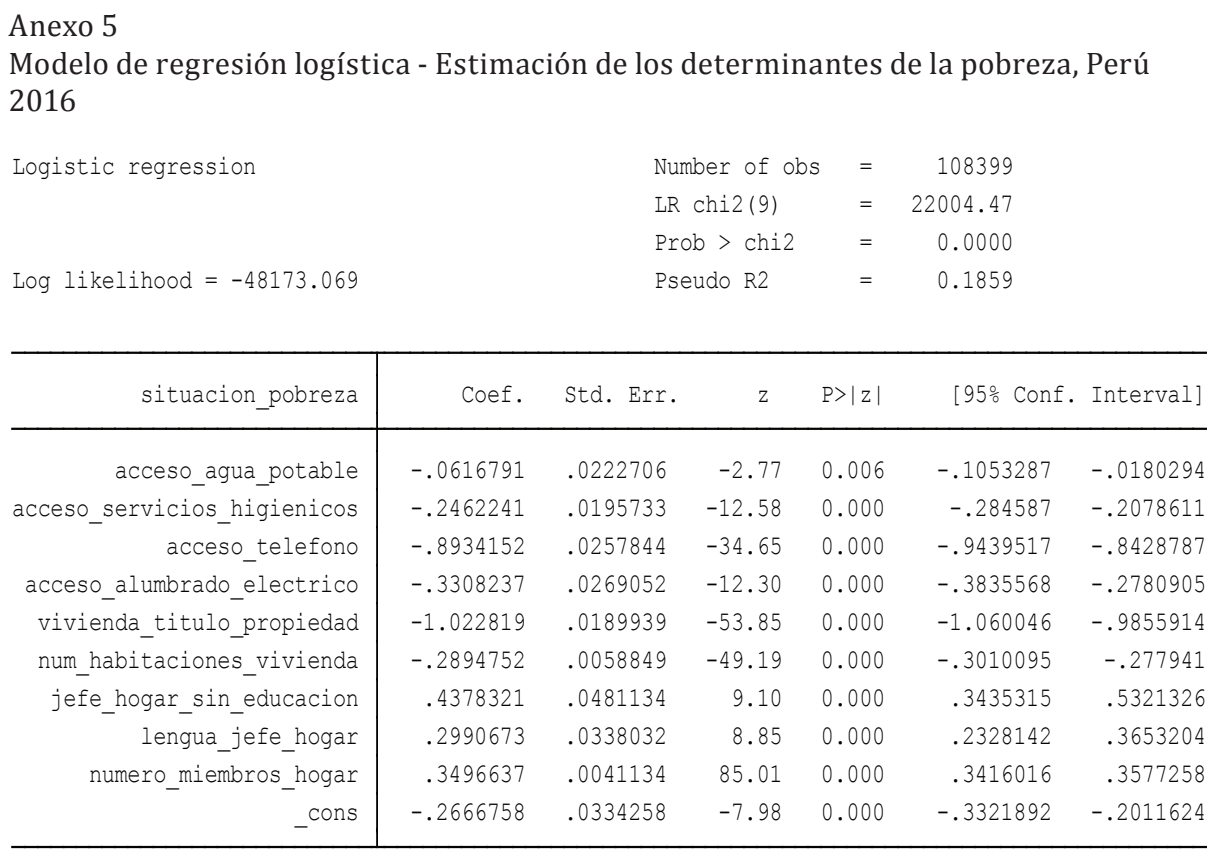

\section{Anexo6}

Modelo de regresión logística - Estimación de los determinantes de la pobreza, Perú 2016, (efectos marginales)

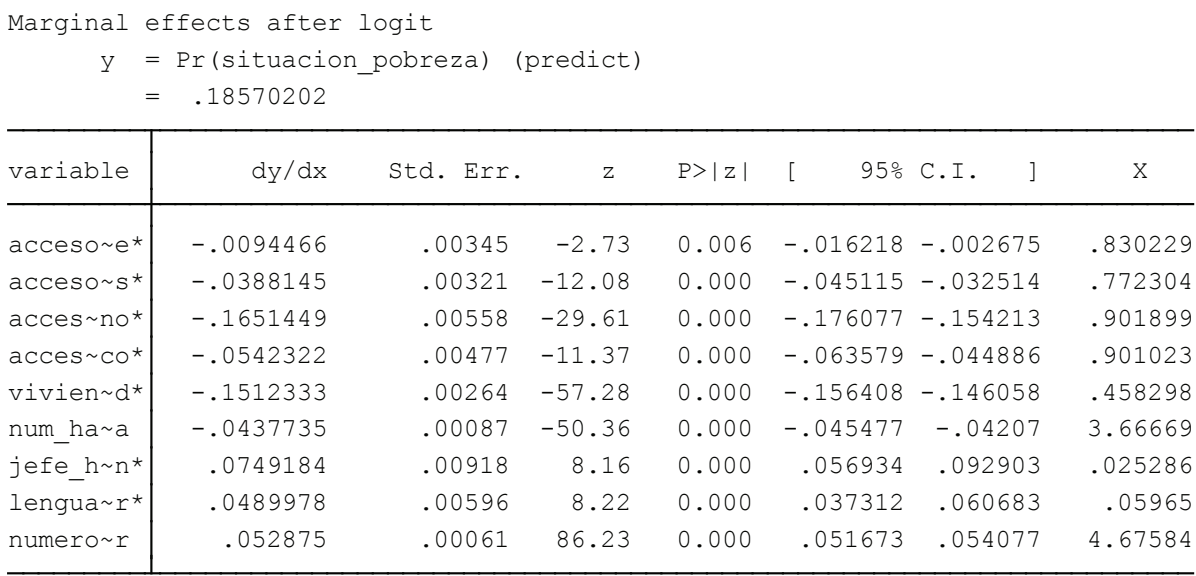

(*) $d y / d x$ is for discrete change of dummy variable from 0 to 1 
